\title{
A photometric and spectroscopic study of the stellar populations in the Large Magellanic Cloud
}

\author{
A. Mucciarelli ${ }^{1}$, F. R. Ferraro ${ }^{1}$, E, Carretta $^{2}$, \\ L. Origlia ${ }^{2}$ and F. Fusi Pecci ${ }^{2}$ \\ ${ }^{1}$ Dipartimento di Astronomia, Università di Bologna, Italy \\ 2 INAF-Osservatorio Astronomico di Bologna, Italy
}

\begin{abstract}
In the last years we started a long-term project devoted to obtain a complete photometric and spectroscopic screening of the stellar populations of the LMC globular cluster system and surrounding fields. The ultimate goals of this project are as follows:

- The definition of reliable and homogeneous cluster metallicity and age scales from high quality, high resolution spectra and color-magnitude diagrams.

- The detailed study of the chemical abundance patterns of iron-peak, alpha-, s- and r-process elements, in order to constrain the star formation and chemical enrichment timescales of both the cluster and field populations and check for possible self-enrichment in the clusters.
\end{abstract}

Keywords. stars:abundances — galaxies: Magellanic Clouds — galaxies: star clusters — techniques: spectroscopic — techniques: photometric

\section{Chemical Abundance analysis}

We present the results of the chemical analysis of giant stars located in four intermediate-age LMC clusters, namely NGC 1651, 1783, 1978 and 2173, and based on UVES@FLAMES high-resolution ( $\mathrm{R} \sim 40000)$ spectra (Ferraro et al. (2006) and Mucciarelli et al., in preparation). The main results are summaryzed in the following:

- We derive $[\mathrm{Fe} / \mathrm{H}]=-0.30 \pm 0.04,-0.35 \pm 0.03,-0.38 \pm 0.03$ and $-0.51 \pm 0.04$ dex for NGC 1651, 1783, 1978 and 2173, respectively. All clusters show a very high homogeneity in their iron content, the overall scatter being $\leqslant 0.07$ dex and entirely accounted for by internal errors.

- Each cluster exhibits a nearly solar $[\alpha / \mathrm{Fe}]$ abundance ratio consistent with the measurements in the LMC disk (Pompeia et al. (2006)), although the overall spread in the latter is relatively large.

- The [iron-peak/Fe] abundance ratios are roughly solar, as in the Milky Way and suggests that the production of these elements well-tracks that of iron.

- The [s-process/Fe] abundance ratios has a bimodal behaviour: the light s-process elements ( $\mathrm{Y}$ and $\mathrm{Zr}$ ) are clearly deficient while the heavy ones (Ba, La, $\mathrm{Nd}$ ) are enhanced with respect to the solar proportion. Also the $[\mathrm{Eu} / \mathrm{Fe}]$ abundance ratio, which is a good tracer of the r-process occurrence, is enhanced in the LMC ([Eu/Fe] +0.30 dex).

These abundance patterns seem to suggest that the chemical enrichment of the LMC occurred on a relatively long timescale, with AGB stars and type Ia SNe being important contributors. 


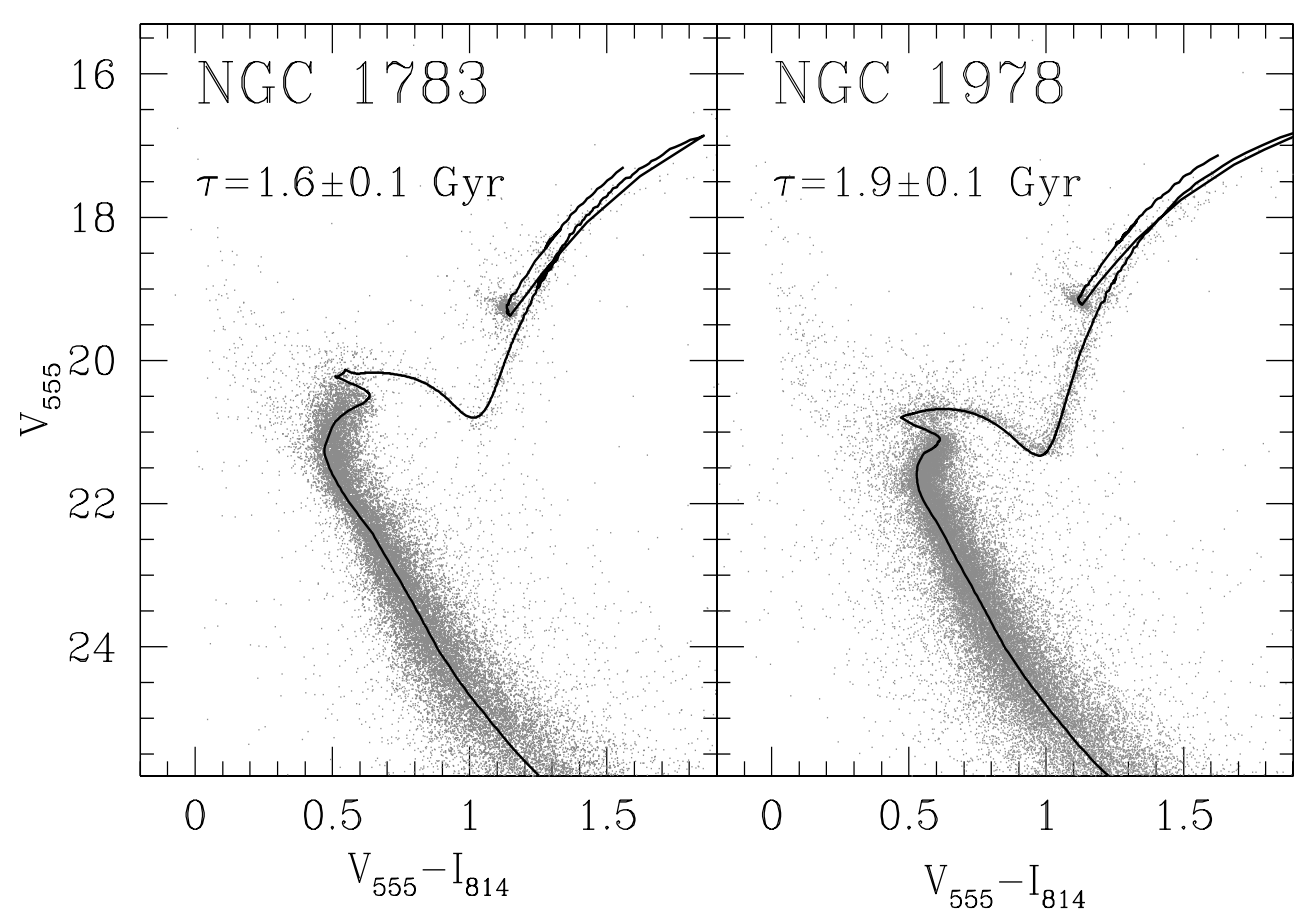

Figure 1. The ACS@HST CMD of the LMC clusters NGC 1783 (right panel) and NGC 1978 (left panel) with overplotted the best-fit isochrones from Castellani et al. (2003).

\section{Towards a new age scale for the LMC clusters}

The precise estimate of the global metallicity $[\mathrm{M} / \mathrm{H}]$ is a key-element in order to derive the correct age of a stellar population. By using the $[\mathrm{Fe} / \mathrm{H}]$ and $[\alpha / \mathrm{Fe}]$ ratios obtained from our chemical analysis, we can obtain a reliable estimate of the global metallicity for any cluster in our dataset. These metallicities, coupled with high-quality HST photometry of the Turn-off region and updated sets of theoretical isochrones, can provide accurate ages for a significant number of pillar LMC clusters to properly define a homogeneous age scale. Here, we present the results for NGC 1783 (in preparation) and NGC 1978 Mucciarelli et al. (2007): in Fig. 1 the ACS@HST CMD for both clusters are reported with the best-fit isochrones. For NGC 1783 the isochrone that best-fit both the morphology and star counts is the Pisa Evolutionary Library (PEL) with a strong overshooting efficiency $\left(\Lambda_{O S}=0.25\right)$ and an age of 1.6 Gyr. For NGC 1978 the best-fit PEL isochrone has a lower overshooting efficiency $\left(\Lambda_{O S}=0.10\right)$ and an age of 1.9 Gyr.

\section{References}

Castellani V., Degl'Innocenti S., Marconi, M., Prada Moroni, P. G., \& Sestito P. 2004, A\&JA 99, 225

Ferraro, F. R., Mucciarelli, A., Carretta, E. \& Origlia, L. 2006, ApJ 643, L33

Mucciarelli, A., Ferraro, F. R., Origlia, L. \& Fusi Pecci, F. 2007, astro-ph-0701649

Pompeia, L., Hill, V., Spite, M., Cole, A., Primas, F., Romaniello, M., Pasquini, L., Cioni, M.-R.\& Smecker-Hane, T., 2006, astro-ph-0604009 